\title{
Effect of health on Nutrition/Dairy Foods and Human Nutrition
}

\author{
Dr. Nita Hitesh Shah*
}

Keywords: Health, Nutrition, Food

\section{INTRODUCTION}

Scientific progress in nutrition, medical and food sciences is having an increasing effect on our approach towards nutrition. There had been a growing awareness about the effects that nutrition has on the health of an individual. Nutritive foods play an important role as an agent for improving health, help in disease prevention and hence maintaining the well being of an individual. Researchers suggest that health of a person depends upon three chief factors; genetic factor, exercise and nutrition.

With the growing researches in food and nutrition, a new class of food known as functional foods has come into being. These functional foods are the nutritive foods that contain certain health-promoting components in addition to the traditionally occurring nutrients. Milk and other dairy products can be identified as one of these kind of functional food as it contains bioactive peptides, probiotic bacteria, antioxidants, highly absorbable calcium, conjugated linoleum acid and other biologically active components. In addition to providing nutrition to the body, these components also alleviate the health of an individual and thus help in prevention of many diseases. The following paper reviews and discusses some of the latest findings regarding the role of milk and other dairy foods in nutrition and health.

\section{The Nutritive Value of Milk}

Milk contains nine essential nutrients, making it a nutrient-rich source of food. Around eight ounce of milk serving per day provides the desired daily value for calcium, riboflavin and other key nutrients like protein, vitamin A, vitamin B12, vitamin D, potassium, phosphorous and niacin. On a food-to-food comparison, milk provides more potassium to the diet than foods with higher potassium content, such as bananas (2.4 percent) or citrus juices (3.9 percent). Overall, milk is a good source of potassium, providing 11 percent of the daily value per serving. Each 8 oz serving of milk provides about 350-400 mg of potassium. However, it is a poor source of iron. Fermentation of milk or the addition of rennet leads to precipitation of insoluble calcium casein ate curds. The milk proteins mainly consist of casein. These proteins are of a high biological value however the limiting factor of milk is that it lacks sculpture containing amino acids as compared to egg proteins. Milk contains $85 \%$ of water and hence forms a good source of fluid and nutrition to the body.

\section{The Positive Effects of Whey}

Whey, a by-product of cheese making, contains lactose, minerals, vitamins, protein, and traces of milk fat. In addition it also contains some part of SNF (solid but not fats). The traditional role of whey proteins in foods has been to provide dietary nitrogen and amino acids to the body.

*Asso. Prof, Dept. of Home Science, Shah K S Arts \& V M Parekh Commerce College, Kapadwanj, Gujarat University, India

(C) 2014 N. Shah; licensee IJIP. This is an Open Access Research distributed under the terms of the Creative Commons Attribution License (http://creativecommons.org/licenses/by/2.0), which permits unrestricted use, distribution, and reproduction in any Medium, provided the original work is properly cited. 
However, much attention is focused on whey proteins owing to its notable content and its form. Not only are the biological value of whey proteins superior to most other proteins, but they also have a high content of sulfur-containing amino acids like cytokine, methionine which support antioxidant functions.

Whey proteins also contain branched chain amino acids which have been established to help minimize muscle wasting under conditions of increased protein breakdown. These proteins having higher concentrations of the branched chain amino acids help to regulate muscle protein synthesis. This increases their potential use for athletes and others aiming to achieve optimal lean muscle mass. Whey components, particularly individual whey proteins, are emerging as highvalue dairy ingredients with potential use in functional foods. New discoveries of the biological functions of dairy ingredients, along with advances in developing economical separation technologies for bioactive components of whey, offer excellent opportunities to function as a rich source of proteins.

\section{The Curing Tendencies of Dairy Foods}

With the formal research scientific evidences, it is confirmed that many chronic diseases such as cancer, osteoporosis, coronary heart disease and hypertension are connected to an unbalanced diet and insufficient nutrition sources. Studies have revealed that individuals consuming higher levels of dairy products, especially low-fat dairy foods, had a significantly lower risk of developing type 2 diabetes. Other research has implied that dairy foods could help prevent insulin resistance, a precursor of type 2 diabetes.

Several recent studies have also suggested that dairy consumption may help control weight and blood pressure and reduce the risks of health problems such as coronary artery disease and gout. Intake of other dairy foods such as milk, cheese, and yogurt provides essential nutrients needed for body maintenance and helps to reduce the risk of major chronic diseases. In addition, intake of milk and other milk group foods such as cheese may reduce children's risk of tooth decay. Intake of dairy foods, because of their calcium content, may help to alleviate symptoms of PMS (premenstrual syndrome) in women, and possibly protect against hearing loss in older adults.

Obesity has been identified globally as a rapidly spreading health disorder. Studies suggest that including $24 \mathrm{oz}$. of fat free or low fat milk every day as part of a reduced-calorie diet may help people lose more weight and burn more fat than cutting calories alone. Daily inclusion of yogurt or cheese in the diet can augment weight and body fat loss which occurs from reduced energy intake. In addition to its beneficial effect on hypertension and also positively affects other risk factors for heart disease, dairy foods significantly reduce blood total and low density lipoprotein (LDL) cholesterol without affecting blood triglyceride levels and to lower blood levels of homocysteine, an amino acid which are linked to increased risk of heart disease and stroke.

According to findings, dairy foods such as milk and especially cheese play a protective role in dental health. Nutrients such as protein, calcium, and phosphorus may contribute to the protective effect of these foods against dental caries milk intake does not promote dental caries and may even have a modest caries-protective effect. Under circumstances when the blood calcium level grows low, it borrows calcium from bones and these shortfall later needs to be replenished with sufficient calcium supply. In the opposite situation, when calcium supply does not reach to a level where the loss can be made good, the bones find it difficult to meet the 
demand of calcium, and this result in to osteoporosis ultimately leading to weakening of bones. Dairy foods prove to be an important source of dietary calcium and it is almost imperative to consume such foods to meet the bodily requirements.

\section{Dairy Food Intake Improves Nutrient Intake}

Intake of milk and milk products (e.g., cheese, yogurt) during adolescence is important to meet calcium needs for building bone mass and strength. Milk and other dairy foods are nutrient-dense foods that supply not only calcium and vitamin D (if fortified), but also other important nutrients such as vitamin B12, riboflavin, and protein. Adequate intake of calcium and calcium-rich foods such as milk, cheese, or yogurt positively affects bone health by increasing bone acquisition during growth, and it also helps in slowing age-related bone loss. Milk, yogurt, and cheese provide a high concentration of many nutrients relative to their energy value. Dairy foods contribute only $9 \%$ of total calories available in the nation's food supply. Yet, these foods naturally provide $72 \%$ of the calcium, $32 \%$ of the phosphorus, $26 \%$ of the riboflavin, $23 \%$ of vitamin A, $20 \%$ of vitamin B12,19\% of protein, and18\% of potassium, along with appreciable amounts of other nutrients.

Milk and other dairy foods are the major contributors of dietary calcium. Without consuming dairy products, it is difficult to meet dietary calcium recommendations. Milk and other dairy foods provide $83 \%$ of the calcium in the diets of young children, $77 \%$ of the calcium in the diets of teenage girls, and between $65 \%$ and $72 \%$ of the calcium in the diets of adults.

Foods naturally containing calcium, in particular foods from the Milk, Yogurt \& Cheese Group, are the preferred source of calcium not only because of their high concentration of calcium, but also because they contain other essential vitamins and minerals. The American Dietetic Association recently stated "the best sources of calcium are dairy foods, since they provide you with calcium, phosphorus, vitamin D and calories. One cup of low-fat, fruit-flavored yogurt or milk provides about $300 \mathrm{mg}$ of calcium"

\section{Functional Foods}

Functional foods are a newer addition to the field of nutrition sciences. They can be generally described as the foods which promote health along with providing basic nutrition. The functional component of such foods affects one or more functions of a human body. Such foods can also be naturally occurring and not always synthetic. The fermented products of milk form such a part of functional foods which contain probiotic bacteria. Such probiotic bacteria have a considerable impact on one's physiological development as well as it also alleviates the intestinal health of an individual. In addition, evidence of health benefits associated with the presence of specific components or bacteria is progressively gaining established scientific credibility. It is therefore understandable that among the best known examples of functional foods are fermented milks and yogurts containing probiotic bacteria.

\section{The Probiotic Dairy Products}

In the year 1908, the Nobel Prize winning Russian scientist, Elie Metchnikoff, linked the long, healthy life of Bulgarian peasants to their high intake of fermented milk products containing lactic acid producing microorganisms. He theorized that the lactic acid bacteria in fermented milk displace undesirable bacteria normally present in the intestine, resulting in a healthier life. 
However it needs to be noted that different strains, species, and genera of bacteria may have unique effects.

The probiotics are such healthy and friendly microorganisms which promote the health of an individual when consumed in a substantial quantity. The majority of probiotics are lactic acid bacteria, especially lactobacilli and bifid bacteria. These probiotic bacteria are almost exclusively found in dairy foods like yoghurt, cultured milk drinks, Sweet Acidophilus, kefir, and some cottage cheese and ice creams. Probiotics also help in regulating the severity of acute diarrhea. It also reduces the chances of colon cancer and also helps strengthen the immune system. The dairy foods, which are a natural vehicle for the probiotics to the intestine, are a desirable source of probiotics. Probiotics such as lactobacilli and bifid bacteria stimulate certain cellular and antibody functions of the immune system, which in turn may increase resistance to immunerelated diseases

\section{Summary}

Milk and other dairy foods have been recognized as important foods since 4000 B.C. On account of dairy foods' natural combination of essential nutrients, these foods have continued to be a key component of diet. On the basis of the evidences presented by the researches, the belief has been established that the inclusion of dairy foods in diet has a powerful influence on health and wellbeing. The food industry has effectively taken this into consideration and additional food items such as flavored yoghurt and low fat and low calorie milk have been introduced to cover this shortfall of nutrition. The use of probiiotics, prebiotics, synbiotics and functional food has been popularized as food products with special characteristics, which alleviate the health.

Because of their unique combination of nutrients, dairy foods cannot be duplicated by a fortified food or dietary supplement. To meet the basic needs of proteins, vitamins and minerals, it becomes imperative to make the dairy foods an important part of one's diet. Milk and other dairy foods contribute to recommended nutrient intakes and promote health throughout life, from infancy through older adult years. Hence, Small dietary changes, such as increasing dairy food intake, can improve the nutritional quality of the diet and play a beneficial role in health promotion and disease prevention. On account of such benefits, the potential use of dairy ingredients in a variety of food products to enhance health has captured the attention of food scientists.

\section{REFERENCE:}

1. Lawson1, Ruth. Moss, Angela. Givens, Ian. 2001., The role of dairy products in supplyingconjugated linoleic acid to man's diet: a review, Nutrition Research Reviews, 14 (3)

2. Lois D. McBean, M.S., R.D., 2001. Health enhancing Properties of Dairy Ingredients., The dairy council digest. 72 (2)

3. Lois D. McBean, M.S., R.D., 2003. Healthy Snacking For Healthy Kids., The dairy council digest. 74 (4) 
4. Lois D. McBean, M.S., R.D., 2004. Dairy Foods' Contribution to Nutrient Dense Diets., The dairy council digests. 75 (1)

5. Lois D. McBean, M.S., R.D., 2004. The Benefits of Dairy Foods In Health Promotion., The dairy council digest. 75 (3)

6. Lois D. McBean, M.S., R.D., 2005, Probiotics: considerations for human health. The dairy council digest. 76 (1)

7. Lois D. McBean, M.S., R.D., 2005. Probiotics: Considerations For Human Health., The dairy council digest. 76 (1)

8. Lois D. McBean, M.S., R.D., 2005. The New Food Guidance System., The dairy council digest. 76 (4)

9. Miller, Gregory D. 1999, The Role of Calcium In Prevention of Chronic Diseases, national dairy council, north Carolina

10. Sanders, M. E. (1998) Overview of functional foods: emphasis on probiotic bacteria. Int. Dairy J. 8: 341-347. 